\title{
Vehicle License Plate Detection and Perspective Rectification
}

\author{
Musaed Alhussein ${ }^{1}$, Khursheed Aurangzeb ${ }^{2, *}$, Syed Irtaza Haider ${ }^{2}$ \\ ${ }^{I}$ Department of Computer Engineering, College of Computer and Information Sciences, \\ King Saud University, \\ Riyadh 11543, Saudi Arabia \\ ${ }^{2}$ College of Computer and Information Sciences, King Saud University, \\ Riyadh 11543, Saudi Arabia \\ kaurangzeb@ksu.edu.sa
}

\begin{abstract}
The character segmentation and perspective rectification of Vehicle License Plate (VLP) is essential in different applications, including traffic monitoring, car parking, stolen vehicle recovery, and toll payment. The character segmentation of the VLP and its horizontal as well as vertical (pan and tilt) correction is a crucial operation. It has considerable impact on the precision of the vehicle identification process. In this work, we investigate an effective framework for the perspective rectification and homography correction of vehicle's images. The captured images of the vehicle could be tilted in vertical or horizontal or verticalhorizontal mix directions due to different movements. For reasonable high identification results, a polynomial fitting based homography correction method for rectifying the tilted VLPs is applied. A method for determining four corner points of the rotated VPLs is explored. These four detected corner points are applied in the homography correction algorithm. For comprehensively evaluating the performance of the proposed framework, the detected VLPs in various directions, such as horizontal, vertical, and mix horizontal-vertical, are rotated. For the experiments, the real images of the vehicles in the outdoor environment, from different directions and different distances are captured. With our proposed method, we achieve an accuracy of $97 \%$ and $95 \%$ for the simulated and real captured images, respectively.
\end{abstract}

Index Terms-Image processing; VLP extraction; Homography correction; Perspective distortion; Smart parking; Smart Cities.

\section{INTRODUCTION}

The constituents of smart cities are smart infrastructure, smart buildings, smart transportation, smart grids, communication technologies, smart governance, and e Healthcare [1]. These individual constituent parts make the cities well-organized as well as smart, and define smart city as a place where daily services and activities are made wellorganized, efficient, and sustainable by utilizing the information and communication technology (ICT). We can also add that the ICT and other advance technologies transform into better services for residents in a smart city.

Manuscript received 7 January, 2019; accepted 12 June, 2019.

The authors extend their appreciation to the Deanship of Scientific Research at King Saud University for funding this work through the research group (No. RG-1438-034).
The smart city applies the latest technologies to advance the quality of citizen's life as well as improves the efficiency of the operational services while at the same time it guarantees that the social, environmental, and economic requirements of the current and coming generations are satisfied.

The key emerging trends for the evolution towards smart cities are Big Data (BD), machine/deep learning, ICT, Internet of Things (IoT), cloud computing, edge computing, smart buildings, and smart grids. The remarkable development in these emerging trends becomes possible because of the progression in hardware/software design methods, communication technologies, computing power, and availability of cheap sensors for collecting BD. Different types of sensors installed in smart cities are used for producing and accumulating the BD. The collected BD can be automatically analysed/visualized by applying the recently developed/explored deep/machine learning methods.

An enhanced transportation system is one of the crucial constituents of smart cities and its effectiveness has a profound effect on social, economic, and environmental factors of the smart cities. It has a deep impact on the lifestyles of the citizens living in the smart cities also. The smart transportation systems, smart traffic monitoring systems, and smart parking monitoring systems are the fundamental research directions [2]-[8].

The smart parking monitoring system not only help in reducing the cost of the parking space management/monitoring and automatic collection of the parking fee, but have a huge impact on reducing the congestion also. By developing and installing smart parking monitoring/management systems, the citizens are going to be able to find empty parking places quickly and efficiently [2]-[5].

The exploration, implementation, and installation of smart traffic monitoring/management system is crucial for reducing congestion in a large city. Many researchers give a substantial attention to the concerns of the traffic administration in large cities. Different related projects are proposed and explored by different societies in different organizations, which aim to resolve traffic congestion problems, such as waiting time at inter-sections, safety, 
reliability, etc. [6]-[8].

The authors in [6] explore an enhance traffic management system for Hong Kong. They aim for attaining optimal traffic control on tunnels, free-ways, and throughout the Hong Kong. Their targeted factors/parameters include the monitoring traffic for avoiding congestion and the examination of data collected. In [7], the authors target different parameters, such as congestion avoidance, traffic monitoring, and data analysis.

The authors in [8] investigate a model for evaluating the effect of various factors, such as safety, jamming, congestion, and the environmental pollution. Their core focus is the improvement of safety intersections in the cities.

In [9], authors investigate, implement, and install a traffic management/monitoring prototype for watching the work zone. In [10], a traffic congestion detection method is explored. The authors apply fuzzy logic for filtering the real time performance. They use density, speed, and the delay at the intersections as their parameters. The simulations results indicate that their investigated method can precisely imitate the road congestion situation.

The aim of different investigations has been focused on reducing environmental pollution, increasing safety of the traveller, and reducing the traveller's stuck time on the road also. That should help not only in smoothing the traffic flow, but could ensure a healthier lifestyle for the citizens living in the advanced cities. The smart transportation system is one of the main constituent parts of the smart cities. The vehicle identification is an essential task in different research applications of the smart transportation systems, including the parking monitoring [2]-[5], traffic monitoring [6]-[8], and automatic toll payments collection system [11]. The efficiency of the mentioned applications is largely reliant on the precision and efficiency of the Vehicle License Plate (VLP) extraction and character recognition system.

The VLP recognition is quite challenging due to many issues, including background noise and VLP rotation in the captured images. There are some reasons behind these noises, such as vehicle motion at the camera-capturing moment, illumination noise, and different VLP formats [2][9].

In vehicle images, the VLP may seem tilted in different directions, including vertical, horizontal, and mix horizontalvertical. These different tilts have substantial effect on the precision of the character segmentation and recognition, which are critical for the vehicle identification. The tilt and rotation correction of the extracted VLP is vital and highly important for the overall vehicle identification system.

The main contribution of this work is to propose and evaluate a framework for sensing with multimedia sensor for the extraction and homography (tilt) correction of the VLP. The aim is to come up with a method for the tilt correction of the VLP using image processing techniques. We consider that there could be large deviations in the background, outdoor illumination, and the location of the VLPs in the captured images. So, for exploring the accuracy and validity of our proposed framework, a database of the captured images is required, which should contain images in the outdoor environment having VLPs with tilts/rotations in various directions. The images of the vehicles are captured with such variations to use them for exploring the accuracy of the proposed framework.

For assessing performance of the proposed framework, a vast database is required. Such database must have images with tilted/rotated VLPs in all possible directions.

We created our own database that comprises images with VLPs rotated in all possible angles and depths. In total, we created 1094 images in this developed database. Additionally, we captured one hundred and fifty real images with various rotations and background illuminations. We used these captured images for the performance verification of the proposed framework.

The rest of the manuscript is organized as follows. This section (Section I) is followed by the related work in Section II. The experimental setup for the VLP extraction and perspective rectification is presented in Section III. The results and detailed discussion are presented in Section IV. Finally, Section $V$ provides the main findings of the paper.

\section{RELATED WORK}

In urban areas, the traffic congestion is a significant issue to be given substantial consideration and it has an austere impact on the lifestyle of the residents and, on the country, economy. Consequently, solving this issue, it has attained considerable attention from academicians/researchers and funding bodies all over the world. In the last decade, many researchers have given substantial devotion to traffic congestion and management in large cities [6]-[8].

The detection and recognition of the license plate is a significant part of modern traffic management systems. It is a core research area, which has attained significant devotion from the researchers over the last decade [12]-[21].The authors in [12] and [13] present a detailed survey on license plate detection and recognition systems.

The authors in [14] explore application-oriented license plate recognition. They suggest to divide license plate recognition into three categories. Their proposed framework is based on number plate detection, character segmentation, and recognition. It is showed that their method achieves better results compared to the other previous results.

The VLP detection method based on a fast vertical edge detection algorithm (VEDA) is developed by the authors in [15]. They use VEDA for the refining the speed of the method and unwanted-line elimination algorithm (ULEA) for supplementing the quality of the image. They determine that VEDA attains better results in terms of correctness, execution time, and complexity.

Morphology based method for license plate detection is proposed in [16]. The authors apply a morphology based method for detecting important contrast features. Then, a recovery method to reconstruct a license plate from its disjointed parts is used. The results prove to be better in terms of robustness and efficiency compared to the other related works from other researchers.

A region and edge based license plate detection method is explored in [17]. The authors use the Sobel operator for determining the vertical edges and morphological filtering for extracting the candidate regions. They achieve better performance in different scenarios.

The inter-correlation based VLP detection method is 
explored in [18]. The color image based algorithms for VLP detection are presented by authors in [19]-[21]. For extracting VLP in the image, the authors in [21] apply color descriptors and geometrical standard. They apply color information to find the license plate among the candidate regions. The strength of their method is proved by executing tests on the images captured in highly complex background.

The neural network (NN) based methods for VLP detection are intended to train classifiers for attaining suitable response to the images containing vehicles. For increasing the precision, the authors in [22] use the gradientbased technique to train $\mathrm{NN}$ and to achieve the global optimum with a good performance. The authors in [23]-[25] consider the VLP detection method based on neural networks also.

The detected VLPs might be tilted in horizontal, vertical or mix horizontal-vertical directions because of either the movements of the vehicle at the capturing time or capturing angle, which needs to be corrected before the VLP recognition. The tilt correction is very central for achieving high correctness in the recognition step. However, this issue gets modest attention from the researchers over the years [26]-[31].

The authors in [26] propose a vehicle tilt correction method for improving the accuracy of the VLP recognition. They explore a method for the correction of the VLP tilt using the line fitting method. For the tilt correction in horizontal direction, their proposed algorithm clusters the coordinates of the letter/number into two classes for fitting a straight line and computes the rotation angle with the horizontal axis. Then, they rotate the whole image using the rotation angle to correct the horizontal tilt. Similarly, they perform vertical tilt correction by, first, finding the rotation angle along $y$-axis and, then, rotating the whole image accordingly.

The authors in [27] apply principal component analysis (PCA) for the tilt correction in VLP images. They arrange the coordinates of letters/numbers into 2D-dimensional covariance matrix. After this, they determine the rotation angle and the feature vector. Then, they rotate the whole image using the determined angle, to achieve the horizontal tilt correction. For the vertical tilt correction, they apply the PCA and line fitting method. In [26] and [27], the authors test the algorithm only on two images.

The authors in [28] discover a homography-based perspective correction method for the VLP tilt correction. Their obtained results show that the rate of perspective correction for the VLP database of motorcycle and automotive are $89 \%$ and $97 \%$, respectively. The computational complexity of their algorithm is high. Moreover, there is a lack of discussion on the extraction of VLP.

The authors in [29] propose an algorithm for homography correction of VLP using planar homography for single and multiple vehicle detection. Their proposed algorithm is tolerant to images with the complex background. The main issue in their method is that the four corner points for the homography correction are selected manually.

In our current work, we propose an efficient framework for the VLP extraction based on applying 2D convolution on the pre-processed images (Fig. 1). After VLP detection, a tilt-correction algorithm for horizontal, vertical, and hybrid tilt, which could possibly occur in the captured images in the real life, is developed.

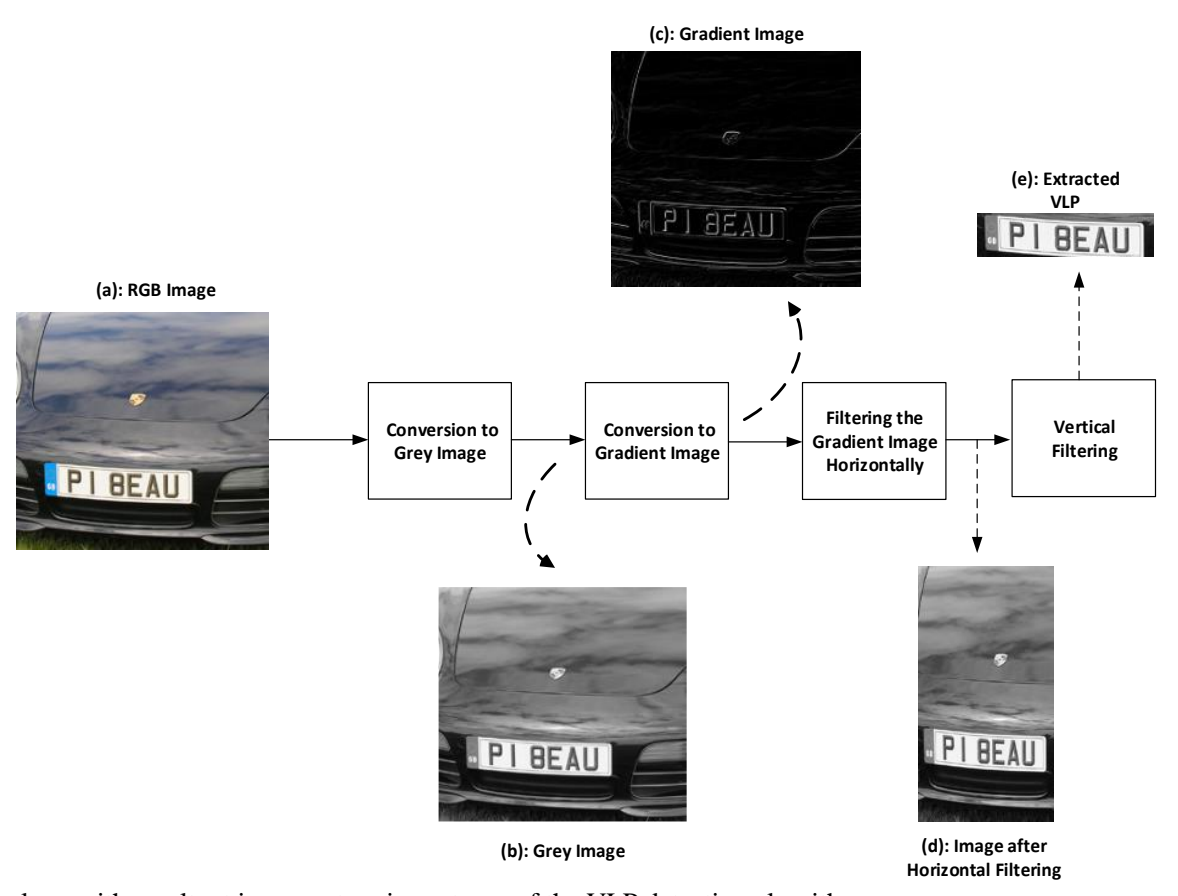

Fig. 1. The labelled tasks along with resultant images at various stages of the VLP detection algorithm.

\section{EXPERIMENTAL SETUP}

Our proposed framework for VLP detection and its tilt correction can be applied in different applications of smart cities, including parking surveillance, monitoring traffic, stolen vehicles recovery, and toll collection. The accurate extraction of the VLP and its tilt correction are central operations for the vehicle recognition. The framework, where 2D convolution and homography for the VLP detection and rotation correction, respectively, are applied, 
is developed. These operations are explained below.

\section{A. The 2D Convolution}

To detect the edges of the VLP in the images, 2D convolution is applied. In Fig. 1(c), we can see that the edges of the digits/letters are brighter.

\section{B. Horizontal Filtering}

First, the gradient image in the horizontal direction is filtered. For horizontal filtering, the convolution of the gradient image with the impulse response of the filter is performed. In the resultant convoluted images, the brightest area corresponds to the VLP. The horizontal edges of the VLP are determined by applying the threshold.

Then, each pixel of the image after the convolution is compared with the selected threshold. In our proposed method, we mark the column index of the first pixel in a row, whose value is greater than threshold. In this way, we find the left edge of the VLP. Similarly, we mark the column index of the first pixel, whose value becomes less than the threshold for determining the right edge of the VLP. Using the horizontal borders of the VLP, its horizontal part is extracted from the image, which is shown in Fig. 1(d).

\section{Vertical Filtering}

For finding the vertical edges of the VLP, the same procedure is applied. The determined vertical and horizontal edges are used to detect the VLP shown in

Fig. 1(e). The interested readers are referred to [30] for the details of the 2D convolution, horizontal filtering, and vertical filtering.

\section{Perspective Rectification}

The image of the vehicle can be captured from many different directions. In many applications, the camera is mounted on a fixed surface usually, but due to the movement and vibrations of the vehicle the VLP in the captured images appears tilted and rotated in various directions. Sometimes the captured images are so much tilted/rotated that the character segmentation and recognition are very difficult to perform. There are mainly three possible tilt ways for the VLP: horizontal tilt, vertical tilt, and horizontal-vertical mix tilt. In this work, we consider $\mathrm{z}$-axis rotation as well as vertical-z axis mix tilt.

For the horizontal tilt, VLP has two possible directions: tilt in the upward direction, when $\alpha>0$ and tilt in the downward direction, when $\alpha<0$ as shown in Fig. 2(a) and Fig. 2(b), respectively. In such kind of images, characters have no shear transform, but a tilt angle between $\mathrm{X}$ and $\mathrm{X}^{\prime}$ axis.

For the vertical tilt, VLP has two possible directions: tilt in the outward direction, when $\beta>0$ and tilt in the inward direction, when $\beta<0$ as shown in Fig. 2(c) and Fig. 2(d), respectively. In such kind of images, the characters have shear transform along the horizontal axis. Fig. 2(e) and Fig. 2(f) show mixed tilt configurations, when $\gamma<0$ and $\gamma>0$.

Perspective correction is an important part of the VLP based vehicle recognition system. The ideal camera perspective is obtained by setting the camera exactly at the front of the VLP. However, such camera setup is not achievable for the real world applications because of the vehicle movement in the front direction. Usually, the camera is fixed at either side of the vehicle that results in capturing non-orthogonal images of the VLP. Consequently, this is going to lead to the lower recognition performance of the systems because of the perspective distortion in the captured non-orthogonal images [29].

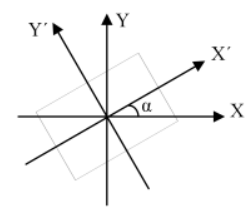

(a)

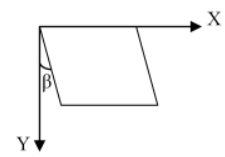

(c)

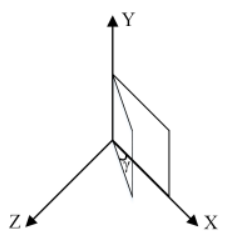

(e)

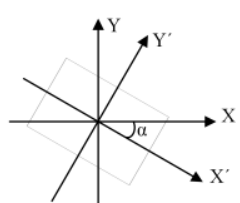

(b)

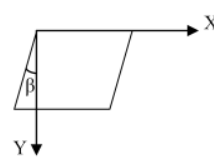

(d)

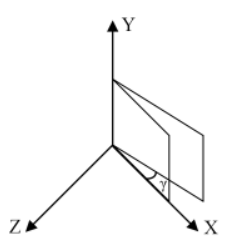

(f)
Horizontal Tilt

Fig. 2. (a) $\alpha>0$; (b) $\alpha<0$; (c) $\beta>0$; (d) $\beta<0$; (e) $\gamma<0$; (f) $\gamma>0$.

The planar homography is a well-known technique, which is usually applied for correcting perspective distortion of the camera. The output of the planar homography is the rectified image of the VLP. The perspective rectification of the nonorthogonally captured images is achieved by finding four corner points of the VLP in these images. Then, the homography matrix is determined, which is used to change the position of every pixel of the image into a specific position on its plane.

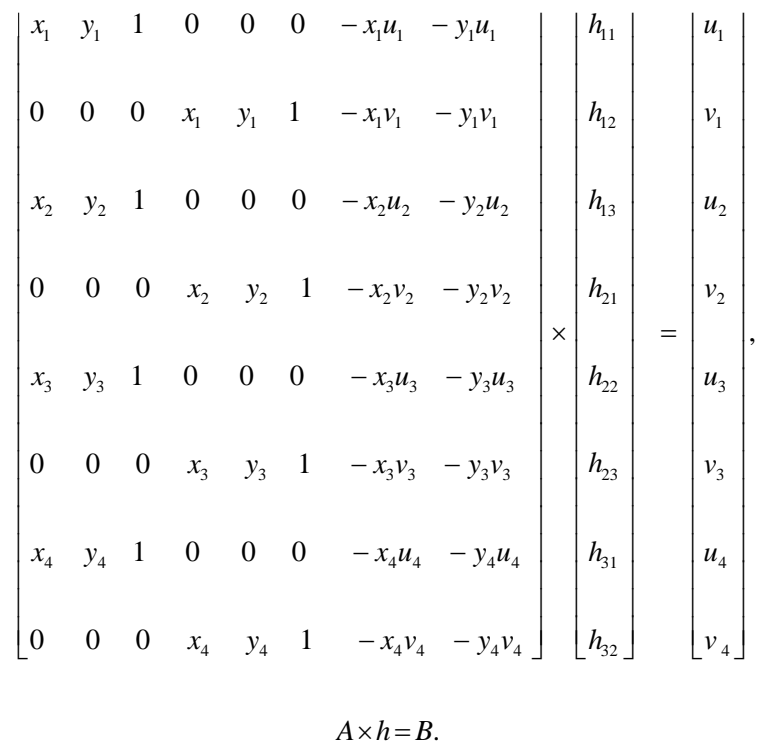

The homography matrix is also called as $2 \mathrm{D}$ to $2 \mathrm{D}$ transformation matrix. The localization of the four corner points of the VLP is necessary for the perspective correction method.

We can calculate the homography matrix by using the expression in (2). Let us consider four corner points of the 
$\operatorname{VLP}\left[\left(\mathrm{x}_{1}, \mathrm{y}_{1}\right),\left(\mathrm{x}_{2}, \mathrm{y}_{2}\right),\left(\mathrm{x}_{3}, \mathrm{y}_{3}\right),\left(\mathrm{x}_{4}, \mathrm{y}_{4}\right)\right]$ that need to be positioned at the desired points $\left[\left(\mathrm{u}_{1}, \mathrm{v}_{1}\right),\left(\mathrm{u}_{2}, \mathrm{v}_{2}\right),\left(\mathrm{u}_{3}, \mathrm{v}_{3}\right),\left(\mathrm{u}_{4}\right.\right.$, $\left.\mathrm{v}_{4}\right)$ ]. By using the homography matrix, we can calculate the new coordinates of the entire image using (3) and (4):

$$
\begin{aligned}
& h=\left(A^{T} A\right)^{-1}\left(A^{T} B\right), \\
& u=\frac{h_{11} x+h_{12} y+h_{13}}{h_{31} x+h_{32} y+1}, \\
& v=\frac{h_{21} x+h_{22} y+h_{23}}{h_{31} x+h_{32} y+1} .
\end{aligned}
$$

\section{RESULTS AND DISCUSSIONS}

Fig. 3 illustrates the framework of the proposed methodology. The algorithm comprises seven stages: (a) preprocessing, (b) extract boundary pixels, (c) detect abrupt changes across the boundary pixels, (d) find two points on each side of the VLP and fit a linear polynomial between these points, (e) detect corner points, (f) correct homography, and (g) crop the final image.

We use the extracted VLP from the convolution based method as an input to the proposed method as shown in Fig. 3(a). First, we binarize the input image and then perform some basic morphological operations to obtain the region of the VLP as shown in Fig. 3(b). White pixels correspond to the foreground (region of interest, i.e., VLP), whereas black pixels correspond to the background. Next, we trace the exterior boundary of the binary image (Fig. 3(c)) and extract the row coordinates of the border pixels. Then, calculate the cumulative sum of the difference between adjacent elements of the row coordinates as shown in Fig. 3(d). The next step is to detect abrupt changes (Fig. 3(d)). The points, where abrupt changes occur, are highlighted with red vertical lines.

In Fig. 3(d), there are three change points, that means we can partition the signal into four different ranges. Each range correspond to each side of the VLP. As shown in Fig. 3(e), for each range, we mark two points using the expressions in (5):

$$
\begin{gathered}
\text { Marked Point }=\text { Mid Point } \pm \text { Difference, } \\
\text { Mid Point }=(\text { Range }(\text { start })+\text { Range }(\text { end })) / 2, \\
\text { Difference }=(\text { Range }(\text { end })-\text { Range }(\text { start })) \times 0.25 .
\end{gathered}
$$

We fit a linear polynomial that draws an infinite line, which passes through each pair of points (Fig. 3(f)). The intersection points between each pair of lines are going to result in four corner points as shown in Fig. 3(g). We pass these corner points to the homography correction algorithm for the implementation of the perspective rectification. The rectified and cropped images are shown in Fig. 3(h) and Fig. 3(i).

Fig. 4 shows the flowchart of the pre-processing steps based on morphological operations. First, we improve the contrast of the input image by applying adaptive histogram equalization technique as seen in Fig. 4(b). Next, we binarize the improved grayscale image by applying global binarization method as shown in Fig. 4(c). Here, we can see that the binary image consists of VLP and noise. Further, we suppress the light structures connected to image border. It is going to reduce the noise around VLP as shown in Fig. 4(d).

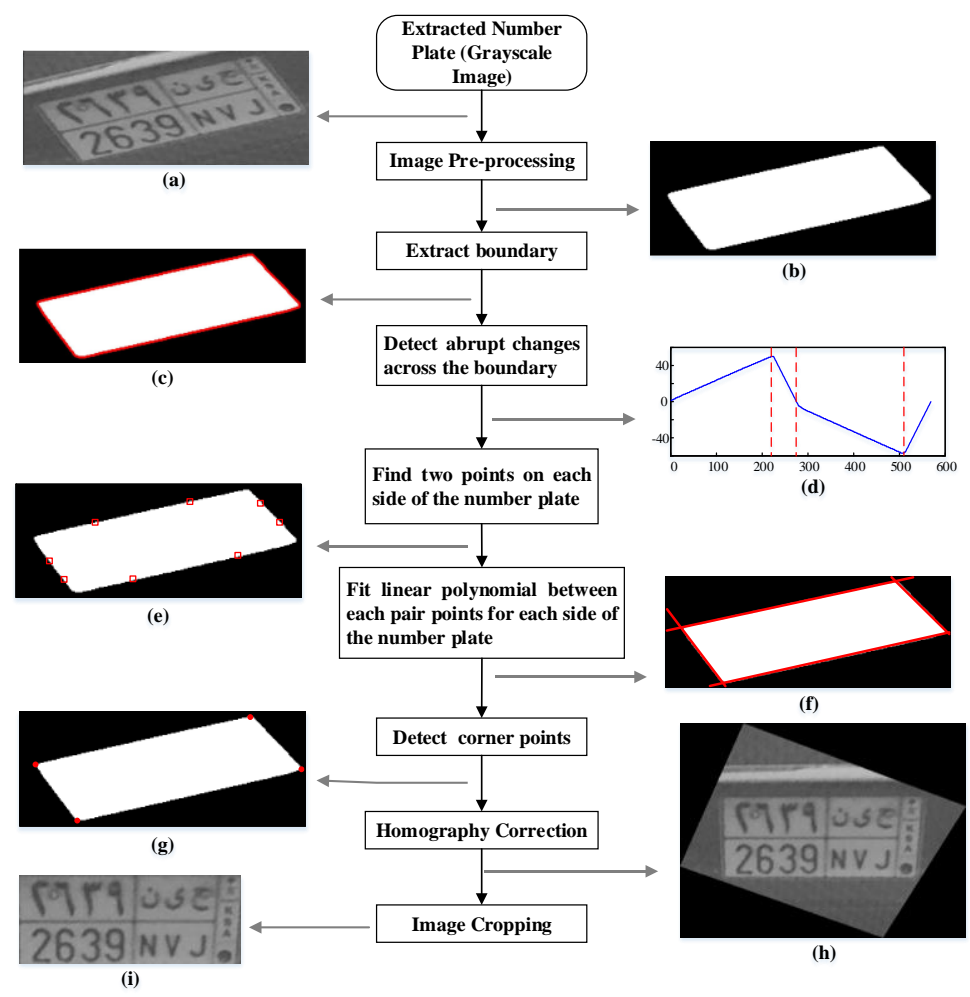

Fig. 3. Algorithmic flow for VLP cropping.

In the next step, we perform flood-fill operation (Fig. 4(e)) on the background pixels, as there are background (black) pixels within the VLP region too. Then, we perform closing operation followed by opening operation with a line and square structuring elements, respectively.

The closing is useful in eliminating small holes and filling 
the gaps (Fig. 4(f)) in the VLP region, whereas the opening opens up a gap between objects as shown in Fig. 4(g). Finally, we generate a convex hull image from the binary image as shown in Fig. 4(h). To suppress the background in order to extract the VLP region for further processing, some basic morphological operations on the input binary image are applied.

The database used in the experiments consists of two sets of VLPs. The first set consists of a single VLP image that is tilted/rotated in various configurations to extensively check the capability of the proposed method. All such configurations and accuracy of the proposed algorithm for the first set of data are mentioned in Table I. All tilted images are rectified using the proposed perspective correction algorithm.

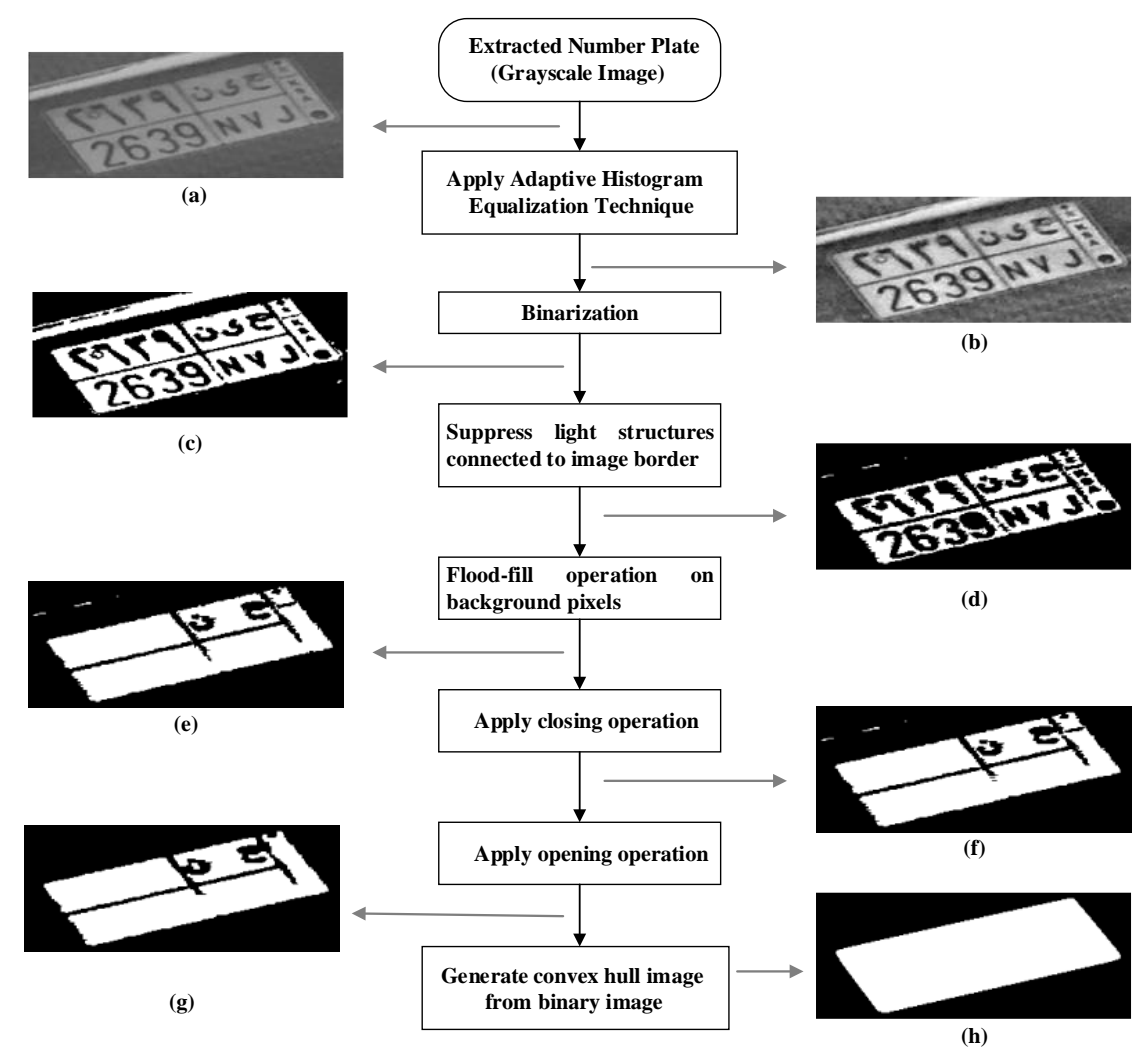

Fig. 4. Algorithmic flow for finding four corner points of the desired VLP.

There are total 14 images for the horizontal tilt as an angle $\alpha$ is varied from $-40^{\circ}$ to $30^{\circ}$ at an interval of $5^{\circ}$. There is no impact on the horizontal tilt by varying the perspective angle $\theta$. All 14 images are correctly rectified. In the vertical tilt, there are total 144 images as an angle $\beta$ is varied from $-60^{\circ}$ to $60^{\circ}$ along with the perspective angle $\theta$ varied from $10^{\circ}$ to $120^{\circ}$. Both angles $\beta$ and $\theta$ are varied at an interval of $10^{\circ}$. All images that fall in the vertical tilt category are correctly rectified.

The third category is depth variation, in which there are total 144 images as an angle $\gamma$ is varied along with $\theta$ at an interval of $10^{\circ}$ from $-60^{\circ}$ to $60^{\circ}$ and from $10^{\circ}$ to $120^{\circ}$, respectively. Out of the 144 images, 141 images are correctly rectified. The horizontal-vertical tilt (mixed tilt) is the fourth category, in which angles $\alpha$ and $\beta$ are varied along with $\theta$. Both angles $\alpha$ and $\beta$ are varied from $-30^{\circ}$ to $30^{\circ}$ at an interval of $10^{\circ}$, whereas $\theta$ is varied from $10^{\circ}$ to $120^{\circ}$. In this category, there are total 432 images, out of which 415 images are correctly rectified. The last category is another type of mixed tilt, in which there are total 360 images as angles $\beta$ and $\gamma$ are varied along with $\theta$.

Both angles $\beta$ and $\gamma$ are varied from $-30^{\circ}$ to $30^{\circ}$ at an interval of $10^{\circ}$, whereas $\theta$ is varied from $0^{\circ}$ to $90^{\circ}$. Out of the 360 images, 349 images are correctly rectified. The overall accuracy of the proposed algorithm for the first set of data is $97.16 \%$.

Table II shows the correctly rectified images, one for each category in the first set along with the three real captured images. Table III shows the images that are classified as incorrectly rectified images by the proposed method. The main reason for almost all incorrectly rectified VLPs is related to the processed binary image that resulted in some portion (edges) of VLP as a background (Fig. 5). This in turn results in incorrect fitting of linear polynomial on one side of the VLP, which leads to the false detection of one of the corners.

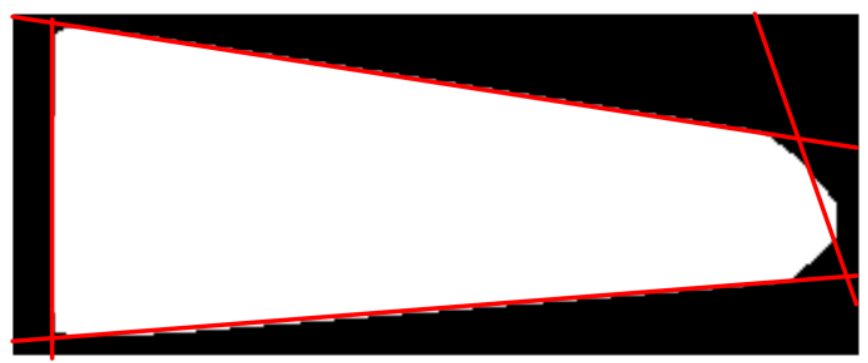

Fig. 5. Processed binary image of Image 1 from Table III.

The algorithm performance is further investigated on the real images captured under various illumination conditions 
for 150 vehicle images taken at different parking facilities in Riyadh, Saudi Arabia. In this set of data, all the captured images represent various configurations listed under the category in Table I. Some captured images have low- contrast, some have stains, and some have shadow on the VLP. Out of 150 images, 143 images are correctly rectified (Table IV).

TABLE I. ACCURACY OF THE PROPOSED METHOD ON SIMULATED IMAGES OF LICENSE PLATE.

\begin{tabular}{|c|c|c|c|c|c|}
\hline Category & Rotation Angle (Degree) & Perspective Angle (Degree) & Total Images & Correctly Rectified Images & Accuracy $(\%)$ \\
\hline Horizontal Tilt & $\alpha=-40^{0}: 5^{0}: 30^{\circ}$ & $\theta=0^{0}$ & 14 & 14 & 100 \\
\hline Vertical Tilt & $\beta=-60^{\circ}: 10^{\circ}: 60^{\circ}$ & $\theta=10^{\circ}: 10^{\circ}: 120^{\circ}$ & 144 & 144 & 100 \\
\hline Depth Variation & $\gamma=-60^{0}: 10^{0}: 60^{0}$ & $\theta=10^{\circ}: 10^{\circ}: 120^{\circ}$ & 144 & 141 & 97.91 \\
\hline $\begin{array}{c}\text { Horizontal } \\
\text { Vertical Tilt }\end{array}$ & $\begin{array}{l}\alpha=-30^{\circ}: 10^{\circ}: 30^{\circ}, \\
\beta=-30^{\circ}: 10^{\circ}: 30^{\circ}\end{array}$ & $\theta=10^{\circ}: 10^{0}: 120^{\circ}$ & 432 & 415 & 96.06 \\
\hline \multirow[t]{2}{*}{$\begin{array}{c}\text { Vertical Depth } \\
\text { Tilt }\end{array}$} & $\begin{array}{l}\beta=-30^{\circ}: 10^{\circ}: 30^{\circ} \\
\gamma=-30^{\circ}: 10^{\circ}: 30^{\circ}\end{array}$ & $\theta=0^{\circ}: 10^{\circ}: 90^{\circ}$ & 360 & 349 & 96.94 \\
\hline & & & 1094 & 1063 & 97.16 \\
\hline
\end{tabular}

TABLE II. PERSPECTIVE RECTIFICATION RESULTS FOR SIMULATED AND REAL CAPTURED IMAGES.

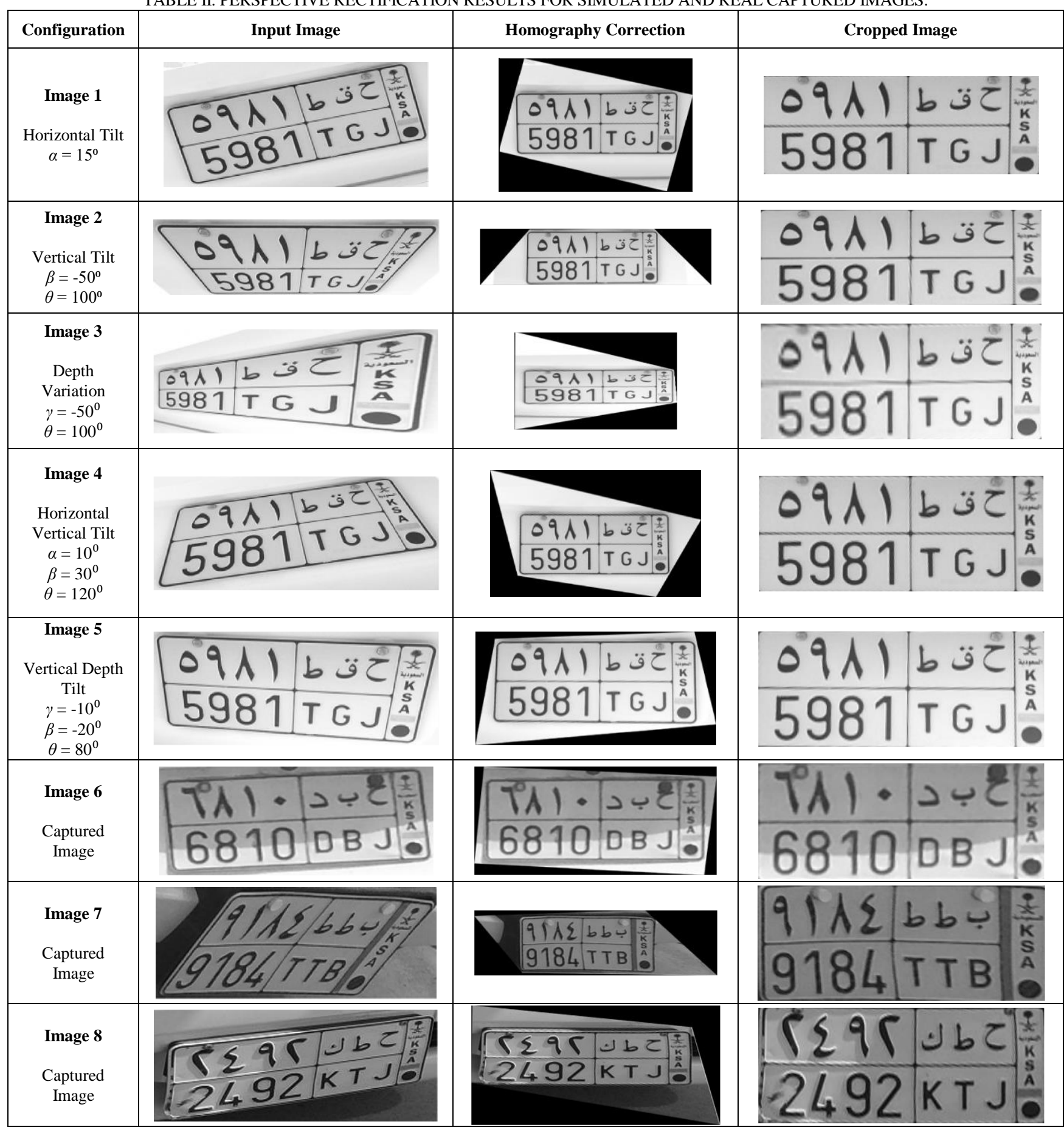


TABLE III. PERSPECTIVE RECTIFICATION RESULTS FOR SIMULATED AND REAL CAPTURED IMAGES CLASSIFIED AS INCORRECTLY RECTIFIED IMAGES BY THE PROPOSED ALGORITHM.

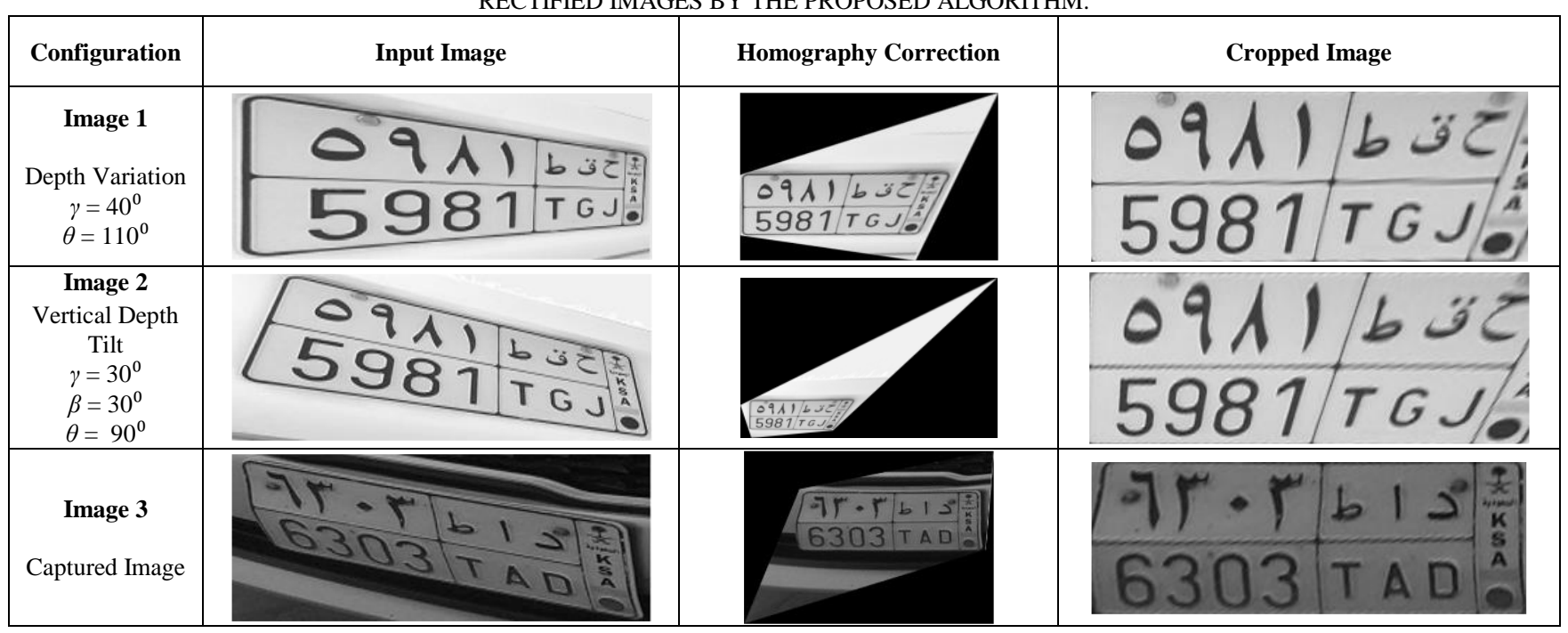

TABLE IV. ACCURACY OF THE PROPOSED METHOD ON CAPTURED IMAGES.

\begin{tabular}{|c|c|c|}
\hline Total Captured Images & Correctly Rectified Images & Accuracy (\%) \\
\hline 150 & 143 & 95.33 \\
\hline
\end{tabular}

\begin{tabular}{|c|c|c|c|c|}
\hline Methods & $\begin{array}{c}\text { VLP } \\
\text { Extraction }\end{array}$ & $\begin{array}{c}\text { Tilt } \\
\text { Correction }\end{array}$ & Contributions & Limitations \\
\hline $\begin{array}{l}\text { Tilt correction based on line fitting } \\
\text { method [26], [27] }\end{array}$ & $x$ & $\checkmark$ & $\begin{array}{l}\text { Horizontal and vertical tilt } \\
\text { correction. } \\
\text { Computationally inexpensive. }\end{array}$ & $\begin{array}{l}\text { Algorithm is tested on two images. } \\
\text { No explanation of VLP extraction. }\end{array}$ \\
\hline $\begin{array}{l}\text { Homography-based perspective } \\
\text { correction method [28] }\end{array}$ & $x$ & $\checkmark$ & $\begin{array}{l}\text { Horizontal/vertical tilt correction. } \\
\text { Tolerant to different lighting, } \\
\text { contrast and size of LPs. }\end{array}$ & $\begin{array}{c}\text { Computational complexity is too high. } \\
\text { No explanation of VLP region } \\
\text { extraction. }\end{array}$ \\
\hline $\begin{array}{l}\text { Perspective rectification using 2D-2D } \\
\text { transformation of planar homography } \\
\text { [29] }\end{array}$ & $\checkmark$ & $\checkmark$ & $\begin{array}{l}\text { Planar homography for single and } \\
\text { multiple vehicle detection. } \\
\text { Tolerant to images with complex } \\
\text { background. }\end{array}$ & $\begin{array}{l}\text { Manual selection of four corner points } \\
\text { for planar homography. }\end{array}$ \\
\hline $\begin{array}{l}\text { Tilt correction using K-L } \\
\text { transformation }[31]\end{array}$ & $x$ & $\checkmark$ & $\begin{array}{l}\text { Horizontal and vertical tilt } \\
\text { correction using K-L } \\
\text { transformation. } \\
\text { High efficiency. } \\
\end{array}$ & $\begin{array}{c}\text { No explanation of VLP region } \\
\text { extraction. }\end{array}$ \\
\hline $\begin{array}{l}\text { Tilt correction based on the straight line } \\
\text { fitting method and projection [32], [33] }\end{array}$ & $\checkmark$ & $\checkmark$ & $\begin{array}{l}\text { Horizontal and vertical tilt } \\
\text { correction using straight line } \\
\text { fitting method. }\end{array}$ & $\begin{array}{l}\text { Problems with images having motion } \\
\text { blur. } \\
\text { Computational complexity is too high. }\end{array}$ \\
\hline Radon transformation based [34] & $x$ & $\checkmark$ & $\begin{array}{c}\text { More robust against various tilt } \\
\text { angles. }\end{array}$ & $\begin{array}{l}\text { Computational complexity is too high } \\
\text { because it needs to calculate the } \\
\text { projection shape of each angle. } \\
\text { No explanation of VLP region } \\
\text { extraction. }\end{array}$ \\
\hline $\begin{array}{l}\text { Gradient features and plate template } \\
\text { based [35] }\end{array}$ & $\checkmark$ & $x$ & More robust against noise and tilt. & $\begin{array}{l}\text { Computational complexity is too high. } \\
\text { Difficult to construct templates of } \\
\text { license plates. Fails due to existence of } \\
\text { the other text. }\end{array}$ \\
\hline Vertical edge matching based [36] & $\checkmark$ & $x$ & More robust against tilt. & $\begin{array}{l}\text { Edges are eliminated by considering it } \\
\text { as noise. }\end{array}$ \\
\hline $\begin{array}{c}\text { Robust license plate recognition } \\
\text { method [37] }\end{array}$ & $\checkmark$ & $x$ & $\begin{array}{l}\text { High efficiency, work on low } \\
\text { contrast and rotated VLPs. }\end{array}$ & Work on fix size LP only. \\
\hline $\begin{array}{l}\text { License plate localization and character } \\
\text { segmentation based [38] }\end{array}$ & $\checkmark$ & $x$ & $\begin{array}{c}\text { Tolerant to images with complex } \\
\text { background, rotation, and low } \\
\text { contrast. }\end{array}$ & Computational complexity is too high. \\
\hline $\begin{array}{l}\text { Automatic license plate location and } \\
\text { recognition [39] }\end{array}$ & $\checkmark$ & $x$ & $\begin{array}{l}\text { Tolerant to different lighting, } \\
\text { contrast, and size of LPs. }\end{array}$ & $\begin{array}{l}\text { Problems with images having complex } \\
\text { background. }\end{array}$ \\
\hline $\begin{array}{l}\text { Efficient embedded NN based LP } \\
\text { recognition system [40] }\end{array}$ & $\checkmark$ & $x$ & $\begin{array}{l}\text { High efficiency, tolerant to low } \\
\text { contrast and different lighting. }\end{array}$ & $\begin{array}{l}\text { Problems with images having complex } \\
\text { background. }\end{array}$ \\
\hline Proposed method & $\checkmark$ & $\checkmark$ & $\begin{array}{l}\text { Automatic selection of four } \\
\text { corner points for planar } \\
\text { homography. } \\
\text { Horizontal, vertical, and hybrid } \\
\text { tilt correction. } \\
\text { Computationally inexpensive. } \\
\text { Robust against various tilt angles. } \\
\text { Tolerant to different lighting, } \\
\text { contrast, and sizes of the VLP. }\end{array}$ & $\begin{array}{l}\text { Problems with images having dented } \\
\text { VLPs. }\end{array}$ \\
\hline
\end{tabular}


In the real captured images dataset, only positive data is used. That means that the dataset consists of driver-view images with distinguishable license plates. The images with unrecognizable VLP or no vehicle at all are not used in this study, which is why the sensitivity and specificity of the proposed method is not presented. Table $\mathrm{V}$ shows the comparison of the proposed method with other related methods. In [29], the authors manually select the corner points for the planar homography. However, in the proposed method, corner points are automatically selected. The only limitation of the proposed method is that the algorithm is not well suited for the damaged/dented VLPs.

\section{CONCLUSIONS}

For VLP extraction in images of vehicles captured in varying conditions, such as different background, different capturing angles, and variable distances between the camera and the vehicle, an algorithm is developed. The VLP detection and its character segmentation/recognition are essential in various applications of the vehicle identification, including smart car parking, traffic monitoring, automatic toll payment, and recovery of stolen vehicles. Both the detection of VLP and its tilt correction are important tasks, which have a considerable impact on the accuracy of the character segmentation and ultimately the vehicle identification. In this work, for the extraction and tilt correction of VLPs, a novel method is implemented and evaluated. For VLP detection and tilt correction, the convolution and the homography based methods are applied. For determining horizontal and vertical borders of the VLP in the images, the horizontal and vertical filtering are applied. These borders are used for extracting the VLPs, which may be tilted in various directions due to the movement of the vehicle at the capturing time. For determining the four corner points of the actual VLP, the morphological operations and polynomial fitting are applied. These corner points are used in the homography equation for correcting the tilted VLPs. The performance of our proposed scheme using 1094 images, in which the VLPs are purposely rotated and tilted in various directions, such as horizontal, vertical, and mix horizontal-vertical, is exhaustively evaluated. The $97 \%$ accuracy on the simulated images is achieved. Additionally, the accuracy of the proposed scheme is verified on 150 captured images in various outdoor environment, such as different background, different VLP formats, and shadow on the VLP due to sun. By applying our proposed method on these real captured images, we achieve an accuracy of $95 \%$.

Data Set: The database of the images used for VLP detection will be provided on request to kaurangzeb@ksu.edu.sa.

\section{REFERENCES}

[1] S. P. Mohanty, U. Choppali, E. Kougianos, "Everything you wanted to know about smart cities: The Internet of Things is the backbone", IEEE Consum. Electron. Mag., vol. 5, no. 3, pp. 60-70, 2016. DOI: 10.1109/MCE.2016.2556879.

[2] S. Lee, D. Yoon, and A. Ghosh, "Intelligent parking lot application using wireless sensor networks", in Proc. of the International Symposium on Collaborative Technologies and Systems, 2008, Irvine, CA., pp. 48-57. DOI: 10.1109/CTS.2008.4543911.
[3] B. Zhang, D. Jiang, F. Wang, and T. Wan, "A design of parking space detector based on video image", in Proc. of Int. Conf. on Electronic Measurement Instruments, 2009, pp. 253-256. DOI: 10.1109/ICEMI.2009.5274579.

[4] T. Nguyen and C. Nguyen, "An approach for building an intelligent parking support system", in Proc. of Symp on Information and Communication Technology, 2014, pp. 192-201. DOI: 10.1145/2676585.2676594.

[5] D. Bong, K. Ting, and K. Lai, "Integrated approach in the design of car park occupancy information system (COINS)", Intl J. of Computer Science, vol. 35, no. 1, pp. 7-14, 2008.

[6] C. N. E. Anagnostopoulos, I. E. Anagnostopoulos, V. Loumos, and E. Kayafas, "A license plate-recognition algorithm for intelligent transportation system applications", IEEE Trans. Intell. Transp. Syst., vol. 7, 2006, pp. 377-392. DOI: 10.1109/TITS.2006.880641.

[7] Hong Kong government. intelligent transport system (ITS). Available [Online]: http://www.roadtraffic-technology.com/projects/hong-kong/

[8] N. Al-Holous, M. Syed Masud, and U. Mohammad, "A multidimensional model for vehicle impact on traffic safety, congestion, and environment", Report No: MIOH UTC TS45, 2012. Available. [Online]: http://mioh-utc.udmercy.edu/research/ts-45/index.htm

[9] M. Bathula, M. Ramezanali, I. Pradhan, N. Patel, J. Gotschall, and N. Sridhar, "Poster abstract: Measuring traffic in short-term construction work zones", in Proc of Int. Conf. on Information Processing in Sensor Networks, 2009, pp. 361-362. DOI: 10.1007/978-3-64202085-8_16.

[10] L. Rui, Y. Zhang, H. Huang, and X. Qiu, "A new traffic congestion detection and quantification method based on comprehensive fuzzy assessment in VANET", KSII Transactions on Internet and Information Systems, vol. 12, no. 1, pp. 41-60, 2018. DOI: 10.3837/tiis.2018.01.003.

[11] A. A. Chapate and D. D. Nawgaje, "Electronic toll collection system based on ARM", Int. Journal of Science, Engineering and Technology Research (IJSETR), vol. 4, no. 1, pp. 46-49, 2015.

[12] S. Du, M. Ibrahim, M. Shehata, and W. Badawy, "Automatic license plate recognition (ALPR): a state-of-the-art review", IEEE Trans. Circuits Syst. Video Technol, vol. 23, no. 2, pp. 311-325, 2013. DOI: 10.1109/TCSVT.2012.2203741.

[13] C. Anagnostopoulos, I. Anagnostopoulos, I. Psoroulas, V. Loumos, and E. Kayafas, "License plate recognition from still images and video sequences: A survey", IEEE Trans. on Intelligent Transportation Systems, vol. 9, no. 3, pp. 377-391, 2008. DOI: 10.1109/TITS.2008.922938.

[14] G. S. Hsu, J. C. Chen, and Y. Z. Chung, "Application-oriented license plate recognition", IEEE Transactions on Vehicular Technology, vol. 62, no. 2, pp. 552-561, 2012. DOI: 10.1109/TVT.2012.2226218.

[15] A. Al-Ghaili, S. Mashohor, A. Ramli, and A. Ismail, "Vertical-edgebased car-license-plate detection method", IEEE Transactions on Vehicular Technology, vol. 62, no. 1, pp. 26-38, 2013. DOI: 10.1109/TVT.2012.2222454.

[16] J. Hsieh, S. Yu, and Y. Chen, "Morphology-based license plate detection from complex scenes", in Proc. of 16th IEEE International Conference on Pattern Recognition, 2002, pp. 176-179. DOI: 10.1109/ICPR.2002.1047823.

[17] M. A. Lalimi, S. Ghofrani, and D. McLernon, "A vehicle license plate detection method using region and edge based methods", Comput. Electr. Eng. vol. 39, no. 3, pp. 834-845, 2013. DOI: 10.1016/j.compeleceng.2012.09.015.

[18] L. Hu and Q. Ni, "IoT-driven automated object detection algorithm for urban surveillance systems in smart cities", IEEE Internet of Things Journal, vol. 5, no. 2, pp. 747-754, 2018. DOI: 10.1109/JIOT.2017.2705560.

[19] X. Shi, W. Zhao, and Y. Shen, "Automatic license plate recognition system based on color image processing", Lecture Notes on Computer Science, vol. 3483. O. Gervasi et al., Eds. New York, Springer, 2005, pp. 1159-1168. DOI: 10.1007/11424925_121.

[20] K. I. Kim, K. Jung, and J. H. Kim, "Colour texture-based object detection: An application to license plate localization", Lecture Notes on Computer Science, vol. 2388. S.-W. Lee and A. Verri, Eds. New York, Springer, 2002, pp. 293-309. DOI: 10.1007/3-540-456651_23.

[21] E. R. Lee, P. K. Kim, and H. J. Kim, "Automatic recognition of a car license plate using colour image processing”, Proc. of IEEE Int'l Conf. on Image Processing, vol. 2, Texas, 1994, pp. 301-305.

[22] G. Sun, C. Zhang, W. Zou, and G. Yu, "A new recognition method of vehicle license plate based on genetic neural network", in Proc. of 5th 
IEEE Conf. on Industrial Electronics and Applications, 2010, pp. 1662-1666. DOI: 10.1109/ICIEA.2010.5515189.

[23] M. Fukumi, Y. Takeuchi, H. Fukumoto, Y. Mitsura, and M. Khalid "Neural network based threshold determination for Malaysia license plate character recognition", in Proc. 9th Int. Conf. Mechatron. Technol, 2005, pp. 1-5.

[24] B. Li, Z. Y. Zeng, J. Z. Zhou, and H. L. Dong, "An algorithm for license plate recognition using radial basis function neural network", in Proc. of the Intl. Symp. on Computer Science and Computational Technology, IEEE Xplore Press, Shanghai, 2008, pp. 569-572. DOI: 10.1109/ISCSCT.2008.272.

[25] M. A. Rafique, W. Pedrycz, and M. Jeon, "Vehicle license plate detection using region-based convolutional neural networks", Soft Computing, vol. 22, no. 19, pp. 6429-6440, 2017. DOI: 10.1007/s00500-017-2696-2.

[26] H. Hao, "An algorithm for vehicle license plate tilt correction based on line fitting method", in Proc. of WASE Int. Conf. on Information Engineering, 2010, pp. 162-166. DOI: 10.1109/ICIE.2010.46.

[27] P. Meisen, Y. Junbiao, and X. Zhenghong. "An approach to tilt correction of vehicle license plate", Mechatronics and Automation. ICMA, pp. 271-275, 2007. DOI: 10.1109/ICMA.2007.4303553.

[28] S.-J. Yang, C. C. Ho, J.-Y. Chen, and C.-Y. Chang, "Practical homography-based perspective correction method for license plate recognition", in Proc. of Int. Conf. on Information Security and Intelligent Control, pp. 198-201, 2012. DOI: 10.1109/ISIC.2012.6449740.

[29] D. P. Sihombing, H. A. Nugroho, and S. Wibirama, "Perspective rectification in vehicle number plate recognition using 2D-2D transformation of planar homography", in Proc. of Int. Conf. on Science in Information Technology (ICSITech), 2015, pp. 237-240. DOI: 10.1109/ICSITech.2015.7407810.

[30] K. Aurangzeb, "An efficient method for extraction of vehicle license plate for various applications in smart cities", ITEE Journal Information Technology \& Electrical Engineering, vol. 7, no. 1, pp. 135-141, 2018.

[31] M.-S. Pan, Q. Xiong, and J.-B. Yan, "A new method for correcting vehicle license plate tilt", International Journal of Automation and Computing, vol. 6, no. 2, pp. 210-216, 2009. DOI: 10.1007/s11633-
009-0210-8.

[32] K. Deb, A.Vavilin, J.-W. Kim, and K.-H. Jo, "License plate tilt correction based on the straight line fitting method and projection", in Proc. of Int. Conf. on Comput. Collective Intell., 2010, pp. 184-193. DOI: 10.1007/978-3-642-16696-9_20.

[33] K. Deb, A. Vavilin, J.-W. Kim, and K.-H. Jo, "Vehicle license plate tilt correction based on the straight line fitting method and minimizing variance of coordinates of projection points", in Proc. of Int. Journal of Control, Automation, and Syst., vol. 8, no. 5, pp. 975984, 2010. DOI: 10.1007/s12555-010-0506-z.

[34] X. D. Jia, W. J. Li, and H. J. Wang, "Novel approach for vehicle license plate tilt correction based on radon transform", Journal of Computer Engineering and Applications, vol. 44, no. 3, pp. 245-248, 2008.

[35] S. Kim, D. Kim, Y. Ryu, and G. Kim, "A robust license-plate extraction method under complex image conditions", in Proc. of 16th Int. Conf. Pattern Recognit., Canada, 2002, pp. 216-219. DOI: 10.1109/ICPR.2002.1047833.

[36] M. Yu and Y. D. Kim, "An approach to Korean license plate recognition based on vertical edge matching", in Proc.of IEEE Int. Conf. Syst. Man. Cybern., 2000, pp. 2975-2980. DOI 10.1109/ICSMC.2000.884453.

[37] T. Naito, T. Tsukada, K. Yamada, K. Kozuka, and S. Yamamoto, "Robust license-plate recognition method for passing vehicles under outside environment", IEEE Trans. Veh. Technol., vol. 49, no. 6, pp. 2309-2319, 2000. DOI: 10.1109/25.901900.

[38] J.-M. Guo and Y.-F. Liu, "License plate localization and character segmentation with feedback self-learning and hybrid binarization techniques", IEEE Trans. Veh. Technol., vol. 57, no. 3, pp. 1417 1424, 2008. DOI: 10.1109/TVT.2007.909284.

[39] Z.-X. Chen, Y.-L. Cheng, F.-L. Chang, and G.-Y. Wang, "Automatic license-plate location and recognition based on feature salience", IEEE Trans. Veh. Technol., vol. 58, no. 7, pp. 3781-3785, 2009. DOI: 10.1109/TVT.2009.2013139.

[40] H. Caner, H. S. Gecim, and A. Z. Alkar, "Efficient embedded neural network-based license plate recognition system", IEEE Trans. Veh. Technol., vol. 57, no. 5, pp. 2675-2683, 2008. DOI 10.1109/TVT.2008.915524. 